\title{
ANÁLISE DA PERCEPÇÃO DOS CURSOS ON-LINE DE SOFTWARES (EXCEL E REVIT) OFERECIDOS DURANTE O PERIODO DE PANDEMIA
}

$$
\text { DOI: 10.37702/2175-957X.COBENGE.2021.3495 }
$$

Cesar Alberto Ruver - cesar@ufrgs.br

Universidade Federal do Rio Grande do Sul

Rua Coronel Massot 214

91910-530 - Porto Alegre - RS

Camila Cristiane Caumo Zonta - zontacamila@yahoo.com.br Universidade Federal do Rio Grande do Sul

Rua Jacinto Gomes 409

90040-270 - Porto Alegre - RS

Felipe Pereira Vergara - felipepereiravergara@hotmail.com

Universidade Federal do Rio Grande do Sul

Rua Germano Hasslocher 99

90160-050 - Porto Alegre - RS

Tainá Garcia da Fonseca - taina.fonseca@hotmail.com

Universidade Federal do Rio Grande do Sul

RUA FELIZARDO 496

90690-200 - PORTO ALEGRE - RS

Franciele Oliveira Rauber - franciele.r98@gmail.com

Universidade Federal do Rio Grande do Sul

Rua Carlos Barbosa 109

94850-230 - Alvorada - RS

Isadora Lemes de Oliveira - isa.lemesdeoliveira@gmail.com

Universidade Federal do Rio Grande do Sul

Rua Francisco Ferrer 355

90420-140 - Porto Alegre - RS

marcelo pasko pereira - marcelopasko@gmail.com

Universidade Federal do Rio Grande do Sul

rua mestre perfeito 220 
95670-000 - Gramado - RS

Katlin Modesto Dorneles - katlinmodestodorneles@gmail.com

Universidade Federal do Rio Grande do Sul

Av. Nazário 3780

92035-000 - Canoas - RS

Vítor Schmidt Teixeira - stvitor1@gmail.com

Universidade Federal do Rio Grande do Sul

Rua Doutor Vicente de Paula Dutra 111

90110-200 - Porto Alegre - RS

Willian Valli Silva - WillianValli99@gmail.com

Universidade Federal do Rio Grande do Sul

Rua Doutor José Ataliba Álvares 105

91751-510 - Porto Alegre - RS

Nathália Fátima da Silva Dias - nathaliafsdias@gmail.com

Universidade Federal do Rio Grande do Sul

Rua Visconde do Cairu 476

93821-056 - Sapiranga - RS

Gabriel Wesendonck Stalschuss - wesendonck.stalschuss@ufrgs.br

Universidade Federal do Rio Grande do Sul

Rua Barão do Rio Branco 81

98700-000 - ljuí - RS

Resumo: O Grupo PET Engenharia Civil da UFRGS, entre suas diversas propostas de melhoria do curso de graduação, tem promovido cursos de software de interesse dos alunos e profissionais da área, dentre os quais pode-se destacar o Excel e o Revit. Estes, tradicionalmente, são ofertados de forma presencial, mas devido a pandemia do corona vírus, tiveram que ser reformulados e ministrados de forma on-line. Este novo contexto ampliou os cursos, transformando-os em cursos do tipo MOOC (massivos, abertos e gratuitos) e também passaram a ser tratados como ações de extensão. Ao final dos cursos é solicitado aos participantes o preenchimento de um questionário de feedback (pré-requisito para recebimento do certificado) de avaliação dos cursos. Assim, o objetivo deste trabalho é mostrar o resultado das avaliações da primeira edição on-line destes cursos, sendo analisados dados, como: (a) alcance e abrangência, (b) socioeconômicos e (c) 
qualidade e aprendizagem. Verificou-se que se ampliou significativamente 0 número de concluintes (da ordem de 160\%). Abrangeu estudantes de ensino médio até profissionais com pós-graduação, inclusive de outros estados e diversos cursos, não ficando restrito aos graduandos de engenharia civil da UFRGS. Do ponto de vista socioeconômico, verificou-se que os participantes possuem uma taxa média de computador/pessoa inferior a um, independentemente da renda familiar e número de pessoas residentes com o participante. A avaliação dos cursos em termos da qualidade do material (videoaulas, exercícios, apostilas) e atendimento foi considerada satisfatória, com conceitos de bom a muito bom e notas que variam de 8 a 10.

Palavras-chave: Cursos On-line. Software Excel e Revit. Avaliação de Cursos 


\section{ANÁLISE DA PERCEPÇÃO DOS CURSOS ON-LINE DE SOFTWARES (EXCEL E REVIT) OFERECIDOS DURANTE O PERIODO DE PANDEMIA}

\section{Introdução}

O Grupo PET Engenharia Civil da UFRGS, desde o ano de 2017, vem entre suas diversas atividades de ensino, de pesquisa e de extensão desenvolvidas para a melhoria do curso de graduação, ministrado os cursos gratuitos de software Excel (planilha eletrônica) e de Revit (desenho CAD e BIM), para a comunidade. Todo o conteúdo e o material didático (apostila e exercícios) são elaborados e estruturado pelos alunos petianos. Além disso, aos participantes dos cursos que cumprirem todos os quesitos (realização das atividades e frequência mínima) recebem um certificado de participação com registro de carga horário, que por exemplo, para o caso dos graduandos de engenharia civil da UFRGS, pode ser utilizado para a conversão em créditos complementares. Os cursos originalmente são realizados de forma presencial, mas devido à COVID-19, as atividades presenciais da UFRGS estão suspensas desde o dia 17/03/2020 (Portaria ํ 2286) (UFRGS, 2020a). Por isso, os cursos passaram a ser ofertados de forma on-line. No formato virtual, ao invés de aulas remotas ao vivo, o grupo optou em fazer a gravação de videoaulas, e disponibilizando-os de forma assíncrona no canal do Youtube (também disponibilizados na plataforma Coursify) aos participantes inscritos. Além disso, vislumbrou-se a oportunidade de ampliar a abrangência dos cursos, transformando-os em cursos de extensão devidamente registrados junto a Pró-Reitoria de Extensão (Prorext) da UFRGS.

Assim, o objetivo deste trabalho é mostrar o conjunto dos resultados e os impactos da realização de cursos de curta duração em formato on-line, no âmbito da extensão, ministrados pelo Grupo PET Engenharia Civil. O trabalho foi elaborado a partir da compilação de dados de uma pesquisa (formulário on-line) quali-quantitativa, realizada junto aos participantes ao final da realização dos dois cursos - Excel e Revit. A pesquisa foi dividida em três categorias: (a) alcance e abrangência dos cursos, visando identificar o público participante em termos de formação escolar e alcance territorial; (b) socioeconômico, visando identificar as condições quanto ao acesso de recursos de informática e renda; e (c) qualidade do curso, visando identificar a percepção quanto ao curso e a forma de aprendizado. Neste contexto, a proposta deste trabalho é avaliar o curso, bem como, obter indicativos do aprendizado adquirido pelos participantes.

\section{REFERENCIAL TEÓRICO}

O advento da pandemia devido ao corona vírus, fez com que grande parte das atividades presenciais fossem interrompidas, atingindo desta forma as atividades de ensino em todos os níveis. A portaria no 2286 (UFRGS, 2020a) e a Portaria no 2291 (UFRGS, 2020b), ambas de 17/03/2020, emitidas pela reitoria de UFRGS, e suas posteriores renovações ${ }^{1}$, suspenderam as atividades presenciais de ensino e institui o trabalho remoto, respectivamente, em todo âmbito na Universidade. Já a Portaria no 343, de 17/03/2020 (BRASIL, 2020a), emitida pelo Ministério de Educação, possibilitou a substituição das aulas presenciais durante o período de pandemia. Neste sentido, todas as atividades presenciais tiveram que ser adaptadas. Isso inclui as atividades dos Grupos PET Engenharia Civil, que tiveram que adaptar suas ações, o que inclui a conversão dos cursos de curta duração presenciais em cursos on-line do tipo MOOC. A adaptação ao ensino remoto ou on-line,

${ }^{1}$ Última Portaria no 1433, de 12/03/2021 (UFRGS, 2021), suspende a atividades presenciais de ensino e institui as atividades remotas em mais 30 dias. 
devido a pandemia, veio de forma repentina, de modo que muitos educadores não estavam adaptados ao Novas Tecnologias Digitais da Informação (NTIC), conforme relatado por Falcão et al (2020) e Rodrigues (2020), impondo um novo contexto educacional.

Dentre as diversas modalidades de cursos on-line, pode-se destacar os MOOC (Massive Open On-line Course), que segundo a definição apresentada por Agonács e Matos (2020), são cursos ministrado em ambientes remotos, livres, e massivos por atingirem grande número de participantes. Ainda segundo os autores, este tipo de curso se distingue da forma tradicional de ensino, pois exige muito da capacidade individual e interesse do aluno. Por requer autorregulação e autodireção por parte dos alunos, é caracterizado por altas taxas de evasão. Agonács e Matos (2020) indicam estudos que a taxa de abandono pode atingir os $87,4 \%$. Adamopoulos (2013) cita que o aumento da taxa de retenção dos alunos passa pela satisfação com os professores, material e exercícios. Já os aspectos relacionados a exigência do curso, grande carga de trabalho e a longa duração (várias semanas), pode ter efeitos negativos. Mondini e Domingues (2018), citam que a retenção em cursos on-line é reconhecidamente baixa. As autoras através da pesquisa realizada, verificaram que a retenção está associada a qualidade dos conteúdos disponibilizados, sistemas amigáveis e prazerosos e capacitações que tornam os usuários confiantes na utilização dos sistemas. Outros pontos de discussão entre os pesquisadores é quanto a efetiva aprendizagem (Holanda e Tedesco, 2017). A partir desta percepção o Grupo PET Engenharia Civil, tem focado a realização dos curso on-line, no sentido de oferecer cursos adaptados a uma realidade tecnológica atual, sendo os cursos compostos de recursos audiovisuais atrativos (videoaulas) e com carga horária reduzida, compatível com a necessidade de aprendizado necessária para a utilização das principais funções dos softwares.

\section{METODOLOGIA}

Com a proposta de dar continuidade as atividades do Grupo PET Engenharia Civil de forma remota, foi proposto a adoção de cursos de software de curta duração no formato on-line (Excel de 10 horas/aula com 13 dias de duração e Revit de 15 horas/aula com 17 dias de duração), de acesso universal, adesão livre, gratuitos e sem critérios mínimos para inscrição, podendo serem caracterizados como MOOC. Todos os cursos do Grupo PET Engenharia civil, são organizados, realizados e ministrados por alunos petianos do curso de engenharia civil da UFRGS. Devido as características dos cursos, os mesmos também podem ser classificados como ações de extensão universitária, sendo cadastrados e aprovados junto a UFRGS, uma vez que são ofertados a toda a comunidade.

Os cursos exigiram uma organização, composta pelas seguintes fases: (a) planejamento, (b) inscrição, (c) realização e (d) avaliação dos cursos. A fase de planejamento consumiu a maior parte do tempo (mais de um mês) e exigiu maior envolvimento, pois comtemplou a revisão das apostilas; gravação e edição das videoaulas; elaboração dos exercícios e desafio (somente para o curso de Revit); preparação da arte e divulgação nas mídias sociais, e-mail e grupos de WhatsApp; e alimentação da plataforma on-line Coursify. O período de inscrições foi de uma semana, sendo realizada através do preenchimento de um formulário on-line do GoogleForms, disponibilizado junto as mídias sociais (Instagram e Facebook). Após as inscrições criou-se grupos de WhatsApp, que serviram para troca de informações, fórum de discussões e contanto com os ministrantes e participantes de cada curso. Além dos grupos de WhatsApp, foi disponibilizado o e-mail do grupo para troca de informações. Para a realização dos cursos, os mesmos foram divididos em módulos, e cada módulo era liberado à medida que os participantes iam avançando nos 
cursos (nos cursos citados todas as aulas foram liberadas juntas). As apostilas e os exercícios foram disponibilizados no formato digital não editável e as videoaulas foram disponibilizadas junto ao Youtube (formato não listado). Para o curso de Revit, teve uma etapa final adicional, compreendendo a realização de um desafio, que consistiu na elaboração de um projeto de uma residência, dentro de parâmetros previamente estabelecidos, sendo os mesmos realizados em grupos. Para a conclusão, os participantes tinham que visualizar todas as videoaulas, realizar os exercícios, entregar o desafio (via email, somente para o curso de Revit) e responder a um questionário, no formato de formulário do GoogleForms, com o feedback sobre o curso. Os resultados destes questionários de avaliação foram categorizados visando analisar e identificar (a) o alcance e abrangência, (b) as condições socioeconômicas e (c) a percepção do curso e do aprendizado por parte dos participantes, que é o objetivo central do presente trabalho. Com a conclusão dos cursos, foi realizada a avaliação final dos cursos, por meio da tabulação das respostas dos formulários, verificação do cumprimento dos requisitos por parte dos participantes para a emissão dos certificados. A avaliação dos cursos culminou na elaboração do relatório, submetido a instância competente da UFRGS, que aprovou as atividades. Além disso, os resultados estão sendo apresentados neste trabalho.

\section{$4 \quad$ RESULTADOS}

\subsection{Alcance e Abrangência dos cursos}

Antes de entrar na análise das respostas dos questionários de avaliação, cabe verificar qual o impacto, em números, dos participantes concluintes, desde a primeira edição dos cursos, realizada em 2017 (tabela 1). Nas edições presenciais do curso de Excel houve uma média de 28 concluintes, enquanto a edição on-line contou com 75 concluintes, correspondendo a um aumento de $165 \%$, embora o número de inscrito ter sido de 208 , chegando a uma evasão de 63,9\%. Já o curso de Revit, na modalidade presencial, o número médio de concluintes foi de 21 , enquanto na edição on-line foi de 54 , que corresponde a um aumento de $157 \%$, embora o número de inscritos tenha chegado a 266 , chegando a uma evasão de $79,7 \%$, sendo este percentual maior atribuído a etapa de desafio do curso. A ampliação do número de concluintes, em última análise está associada a modalidade de ensino adotada (on-line e assíncrono) que permite que os participantes realizem as atividades a qualquer tempo (dentro das datas de início e fim de curso), qualquer horário e lugar (desde que tenha internet e um computador com o software instalado), em detrimento ao curso presencial que fica restrito dentro do ambiente da UFRGS e em horário que é concomitante aos horários de trabalho, estágio ou aulas. Outro aspecto que pode ter contribuído para a ampliação do número de concluintes foi o forte trabalho de divulgação, mais impactante visualmente e iterativo junto as mídias sociais, como o Instagram.

Tabela 1 - Número de concluintes dos cursos

\begin{tabular}{cccccc}
\hline \multirow{2}{*}{ Ano } & \multicolumn{5}{c}{ Participantes Concluintes } \\
\cline { 2 - 5 } & \multicolumn{4}{c}{ Excel } & \multicolumn{3}{c}{ Revit } \\
\cline { 2 - 5 } & Presencial & On-line & Presencial & On-line \\
\hline 2017 & 27 & - & 22 & - \\
\hline 2018 & 21 & - & 21 & - \\
\hline $2019 / 1$ & 36 & - & - & - \\
\hline $2019 / 2$ & 29 & $75\left(208^{*}\right)$ & - & $54\left(266^{\star}\right)$ \\
\hline 2020 & - & & & \\
\hline
\end{tabular}

* Número de inscritos 
Figura 1 - Percentual de participantes por estado da federação: (a) curso de Excel e (b) curso de Revit

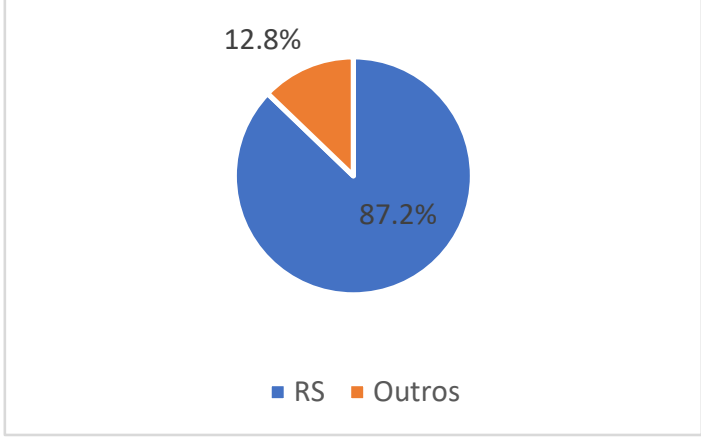

(a)

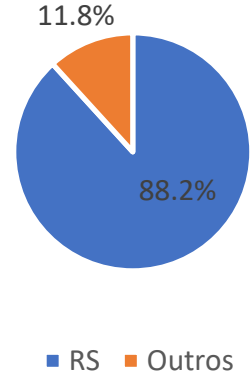

(b)

Embora o Campus Central da UFRGS seja localizada em Porto Alegre, no estado do Rio Grande do Sul, os cursos foram realizados de forma remota e on-line, permitindo que tenham um alcance global. Neste sentido, a figura 1 mostra que houve participantes de outros estados da federação, além do RS, que corresponde a 12,8\% (dois participantes de MG, e um participante por estado de AC, AL, CE, DF, PE, PR, RR e SP) e $11,8 \%$ (dois de participantes de $A L$, e um participante por estado de AP, CE, DF, MG, PB e PI), dos participantes, respectivamente para os cursos de Excel e Revit. Isso mostra, que os cursos on-line conseguem atingir um maior alcance territorial, em relação aos cursos presenciais.

Figura 2 - Percentual de participantes por escolaridade: (a) curso de Excel e (b) curso de Revit

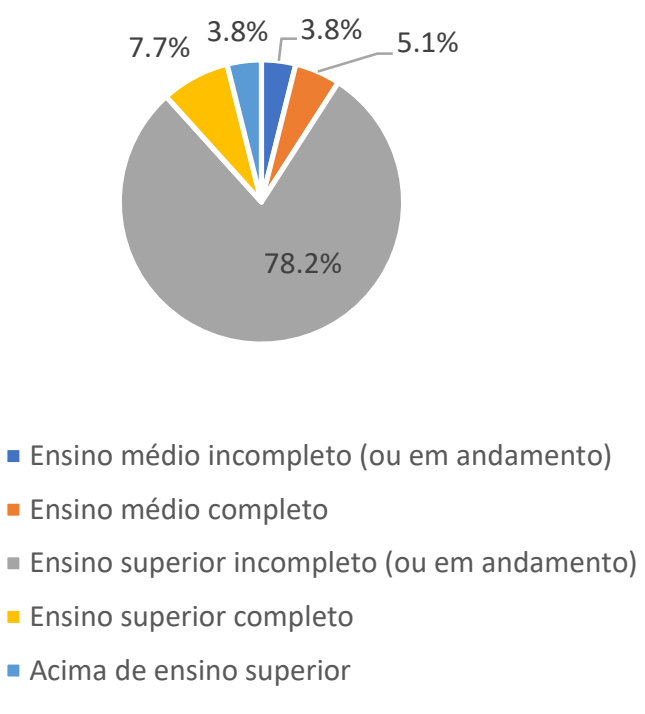

(a)

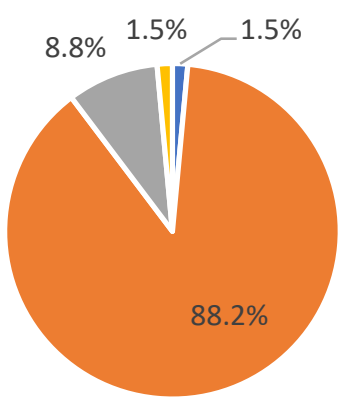

- Ensino médio completo

- Ensino superior incompleto (ou em andamento)

- Ensino superior completo

- Acima de ensino superior

(b) 
Figura 3 - Percentual de participantes com graduação em andamento por tipo de instituição de ensino superior (IES): (a) curso de Excel e (b) curso de Revit

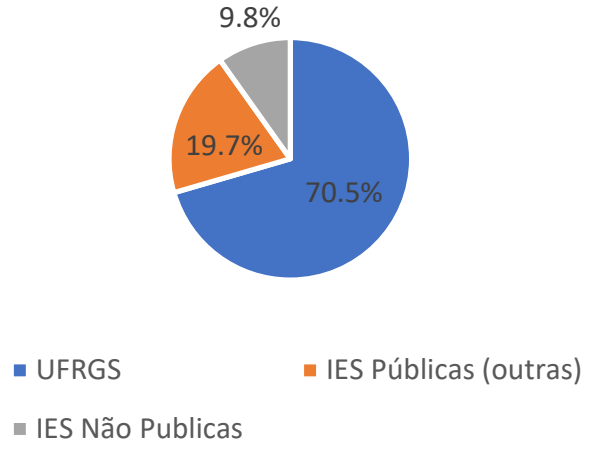

(a)

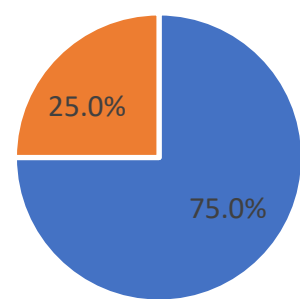

- UFRGS - Outras

Embora não houvesse nenhuma restrição de escolaridade para os participantes dos cursos, verifica-se que a grande maioria são alunos de graduação (figura 2). O Excel por ser um curso mais geral teve a participação de 8,9\% (7 participantes) do com nível médio (3 completo e 4 incompleto), enquanto o curso de Revit contou com 1,5\% (um participante) com nível médio completo. Já a participação de profissionais formados, os percentuais são próximos, com 11,5\% (6 com ensino superior e 3 acima do superior) e 10,3\% (6 com ensino superior e 1 acima do superior), respectivamente, para os cursos de Excel e Revit. Para os participantes do curso de Excel, que são graduandos, verifica-se que $90,2 \%$ são de universidades públicas federais, dos quais $70,5 \%$ são da UFRGS e 11,5 são da UFSM ( 7 participantes) e $8,2 \%$ de outras universidades públicas federais localizadas em outros estados. Em relação a este dado, é interessante observar que os graduandos de outros estados, que fizeram o curso de Excel, são alunos de universidades públicas federais, exceto um participante. Para o curso de Revit, a questão sobre a instituição de ensino na qual os participantes graduando estudam não foi perguntada, mas foi possível obter o percentual dos alunos que estudam na UFRGS, que corresponde a 75\%, em detrimento aos outros $25 \%$ que estudam em outras instituições de ensino superior. O percentual de participantes que são discentes da UFRGS era naturalmente esperado, por tratar-se de um curso promovido pelo Grupo PET Engenharia Civil, em nome da UFRGS.

Figura 4 - Percentual de participantes por curso de graduação, em andamento: (a) curso de Excel e (b) Revit

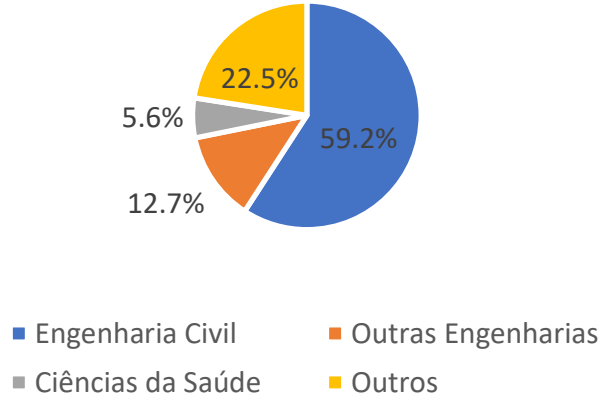

(a)

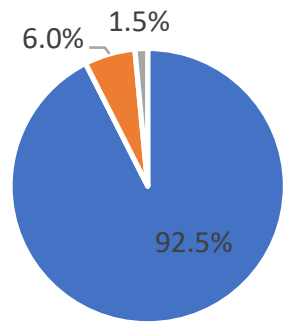

- Engenharia Civil

- Arquitetura e Urbanismo

(b)

A figura 4 mostra os percentuais de participantes em relação ao curso de graduação (em andamento ou concluído). Para o curso de Excel é possível perceber que 59,2\% (42 participantes) são do curso de engenharia civil, $22,5 \%$ são de outras engenharias 
(cartográfica, controle e automação, energia, minas, materiais, mecânica e metalúrgica), totalizando $81,7 \%$ de estudantes e profissionais da área da engenharia; $5,6 \%$ dos cursos de ciências da saúde (medicina, odontologia e psicologia) e 12,7\% de outros cursos (exatas e ciências sociais). Já o curso de Revit foi cursado por $92,5 \%$ (62 participantes) do curso de engenharia civil, 6,0\% (4 participantes) do curso de arquitetura e urbanismo, e um participante de outro curso superior não especificado. Os dados de escolaridade e curso de graduação mostram que o curso de Excel possui uma maior abrangência de público, pois é um software - planilha eletrônica - com uma grande gama de aplicação; enquanto o curso de Revit abrange um público mais específico, por tratar-se de um software de BIM e desenho em ambiente CAD, com aplicação mais restrita ao campo da engenharia civil e arquitetura e urbanismo.

\subsection{Avaliação Socioeconômica}

A figura 5 mostra o número de pessoas que residem com o participante dos cursos. Em relação ao curso de Excel, verifica-se que o maior percentual dos participantes mora com mais uma pessoa (30,8\%), enquanto para o curso de Revit os participantes com mais duas pessoas (32,\%). A figura 6 mostra o número de computadores (tipo PC ou notebook) que os participantes têm disponíveis em casa e em condições para a instalação e utilização dos softwares. Em relação ao curso de Excel, verifica-se que o maior percentual corresponde a dois computadores $(37,2 \%)$ por resistência dos participantes, sendo a que pergunta não especifica o número de computadores acima de três unidades; enquanto para o curso de Revit o maior percentual corresponde a um computador (39,7\%) por residência, sendo perguntado o número de computadores, sem limitação. Ainda, perguntou-se qual a faixa de renda familiar (soma da renda de todas as pessoas que residem com 0 participante), sendo os dados apresentados na figura 7, sendo a pergunta para o curso de Excel ajustada em função do valor do salário mínimo atual (MP no 919/2020) (BRASIL, 2020b). Em relação ao curso de Excel, verifica-se que o maior percentual de renda foi entre $R \$ 4.001,00$ e $R \$ 9.000,00$ (32,1\%), enquanto para o curso de Revit, o maior percentual de renda foi entre $R \$ 4.180,01$ e $R \$ 10.450,00$ (4 e 10 salários mínimos) (47,1\%), sendo ambos dentro da mesma faixa de renda.

Os dados socioeconômicos, apresentados nas figuras 5 a 7, foram cruzados para um melhor entendimento. As figuras 8 e 9, mostram uma relação de tendência de variação entre as informações de renda, número de computadores e número de pessoas residente com o participante, respectivamente, para os cursos de Excel e Revit.

Figura 5 - Quantidade de pessoas que residem com os participantes dos cursos de: (a) Excel e (b) Revit

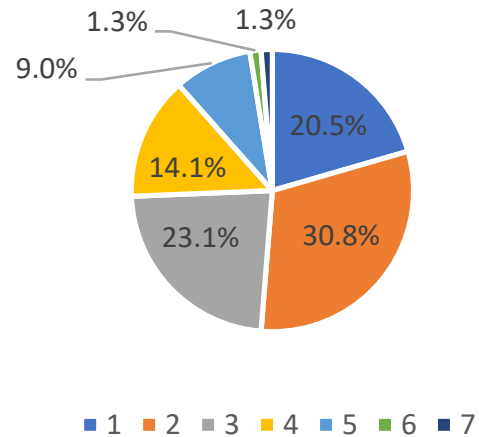

(a)

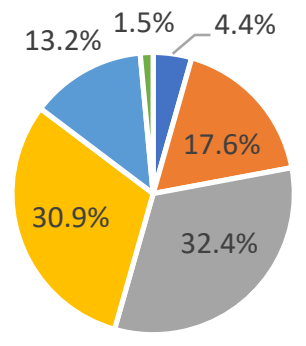

- $1=2-3=4=5-6$ 
Figura 6: Quantidade de computadores tipo PC/notebooks que os participantes possuem em casa em condições para realizar os cursos: (a) Excel e (b) Revit

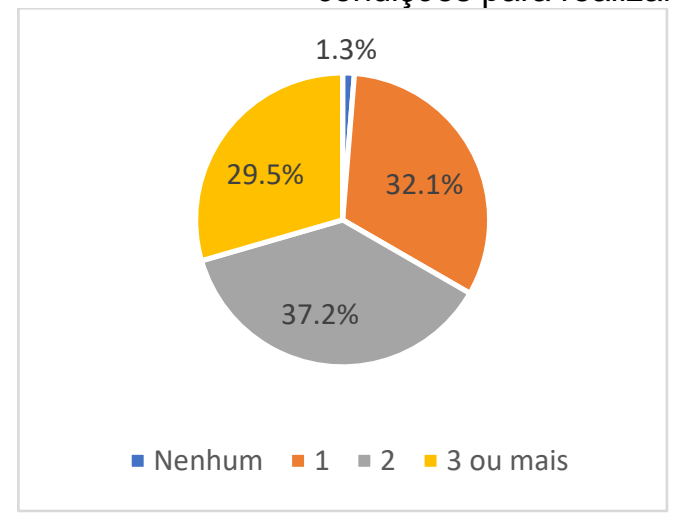

(a)

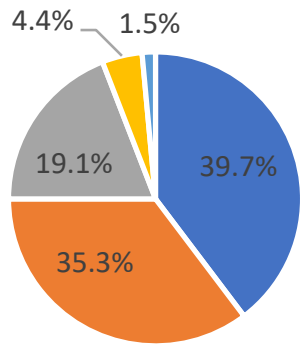

- $1=2-3-4=5$

Figura 7 - Renda mensal familiar dos participantes do curso de: (a) Excel e (b) Revit

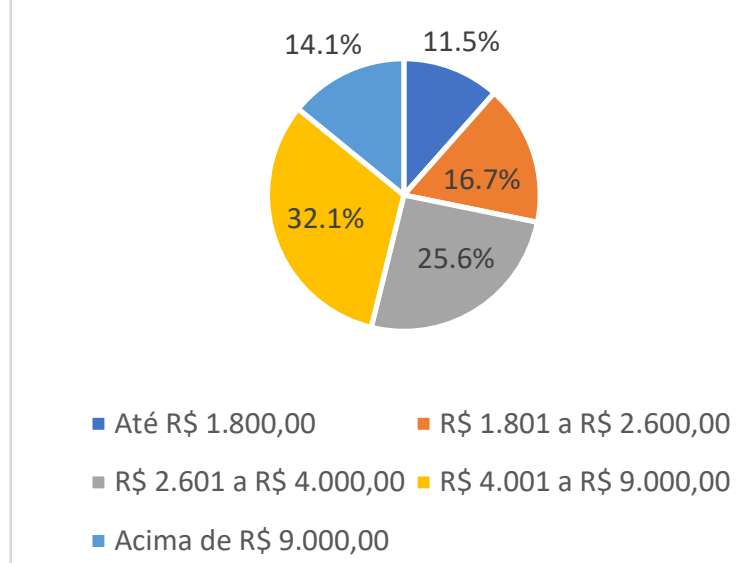

(a)

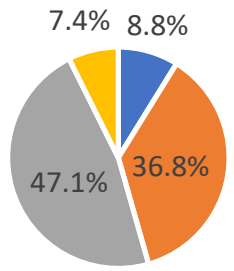

- Até R\$ 2.090,00

. $\mathrm{R} \$$ 2.090,01 a $\mathrm{R} \$$ 4.180,00

v $\mathrm{R}$ 4.180,01 a $\mathrm{R} \$ 10.450,00$

- $\mathrm{R} \$ 10.450,01$ a $\mathrm{R} \$ \mathbf{2 0 . 9 0 0 , 0 0}$

(b)

Nos gráficos 8 e 9 são mostrados todos os dados (número de residentes e computadores) em faixas verticais, sendo que a faixa mais espessa corresponde a um maior número e a mais fina corresponde a uma menor quantidade de dados. Também é mostrada uma linha média de tendência, do número de computadores e número de pessoas que moram com os participantes, em função da renda familiar. A partir dos dados é possível verificar que os participantes do curso de Excel possuem em média 0,89 computadores/pessoa, entre os valores máximos de 3 e nenhum computador/pessoa. Já para o curso de Revit a média é de 0,63 computadores/pessoa, entre os valores máximos de 2 e 0,2 computadores/pessoa. Estes dados mostram que nas residências dos participantes dos cursos, a média de computadores é inferior a um computador por pessoa. Analisando os extremos percebe-se que um dos participantes do curso não possui computador em casa, reside com mais uma pessoa e tem renda de até $R \$ 1.800,00$. No outro extremo, houve um participante, que mora sozinho, possui 3 computadores e renda superior a $\mathrm{R} \$ 9.000,00$. 
Figura 8 - Cruzamento da renda familiar com o número de pessoas residentes e número de computadores para o curso de Excel

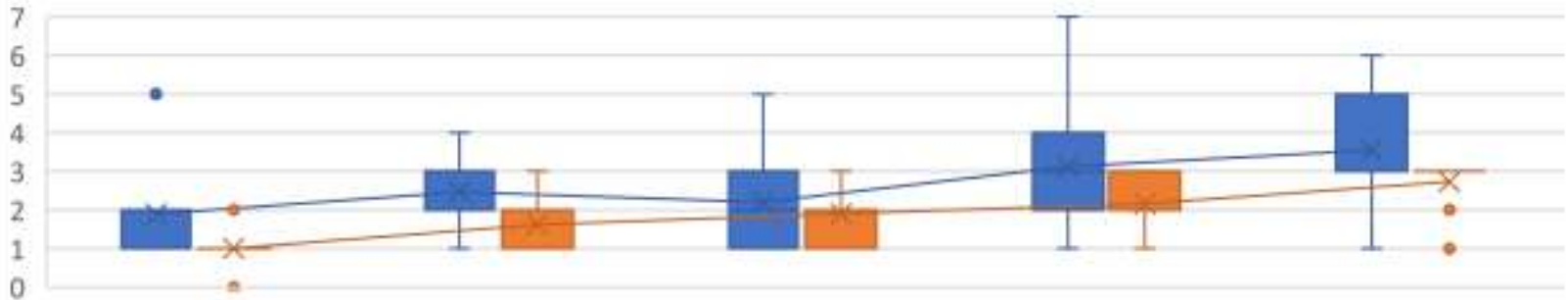

R\$4.001 atè $R \$ 9.000,00$

Até R\$ $1.800,00$

$R \$ 2.601$ ate $R \$ 4.000,00$

Acima de $\mathrm{R} \$ 9.000,00$

Pessoas PC/Notebook

Figura 9: Cruzamento da renda familiar com o número de pessoas residentes e número de computadores para o curso de Excel

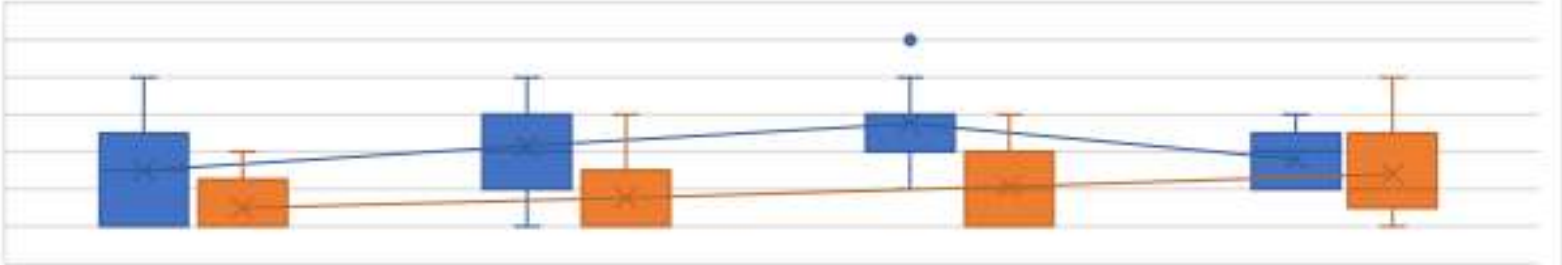

$\mathrm{R} \$ 2.090,01$ a $\mathrm{R} \$ 4.180,00$

Até R\$ 2.090,00

R\$ 4.180,01 a RS $10.450,00$

R\$ $10.450,01$ a $R \$ \ldots$

Pessoas $\mathrm{PC} /$ Notebbok

Pela figura 8 é possível verificar que para os participantes do curso de Excel há uma tendência de crescimento linear do número de pessoas residentes e do número de computadores, em função da renda, sendo o número de computadores inferior ao número de computadores independentemente da renda. A relação de computadores/pessoa é maior entre uma faixa de renda entre $R \$ 2.601,00$ a $R \$ 4.000,00$. Para o curso de Revit (figura 9), também se percebe uma tendência linear de crescimento do número de computadores em função da renda. Para o número pessoas residentes com os participantes, também há uma tendência de crescimento linear com a renda, porém com uma taxa maior, exceto para a faixa de renda mais elevada, onde a taxa de computadores/pessoa é a maior e se aproxima de um.

\subsection{Percepção do Curso}

As figuras 10 e 11 mostram a forma como os participantes ficaram sabendo do curso. Pelas figuras 10 e 11 percebe-se que a maior parte (57,7\% para o Excel e $69,1 \%$ para o Revit) foi via Instagram, sendo está também a principal mídia social alimentada pelo grupo PET Engenharia Civil, que conta atualmente com 2.091 seguidores. Outra forma, foi a rede de contatos formada entre os alunos do curso de engenharia civil da UFRGS (amigos ou grupos de WhatsApp), o que ratifica a participação massiva destes nos cursos. 
Figura 10 - Forma como o participante ficou sabendo sobre a realização do curso de Excel

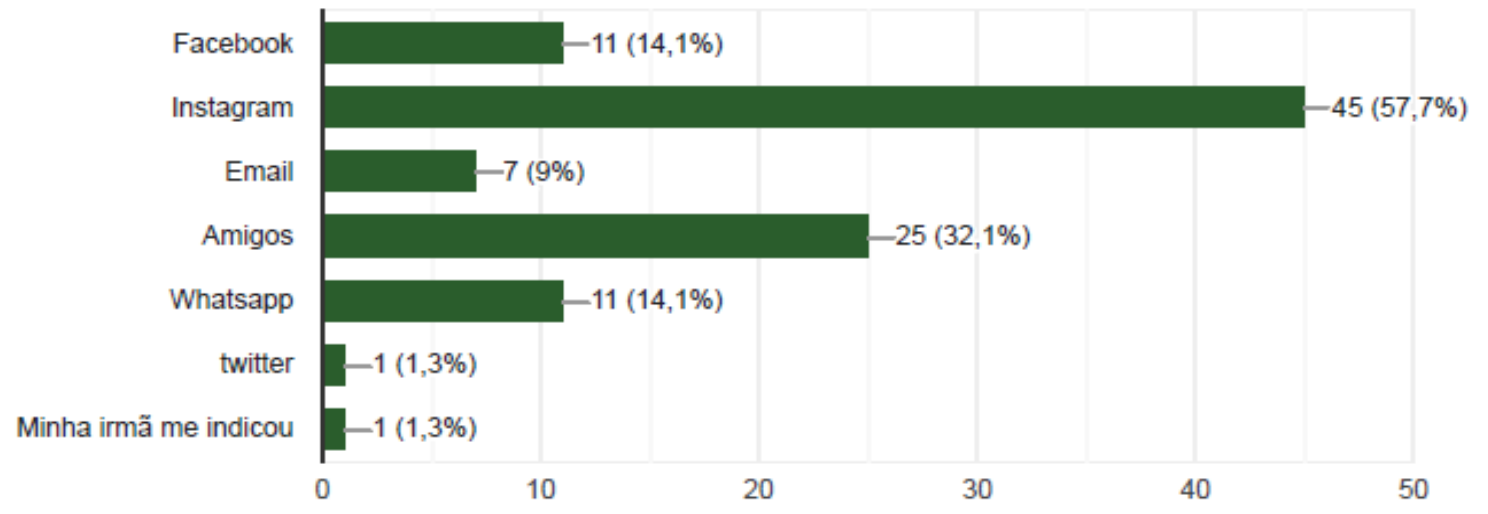

Figura 11 - Forma como o participante ficou sabendo sobre a realização do curso de Revit

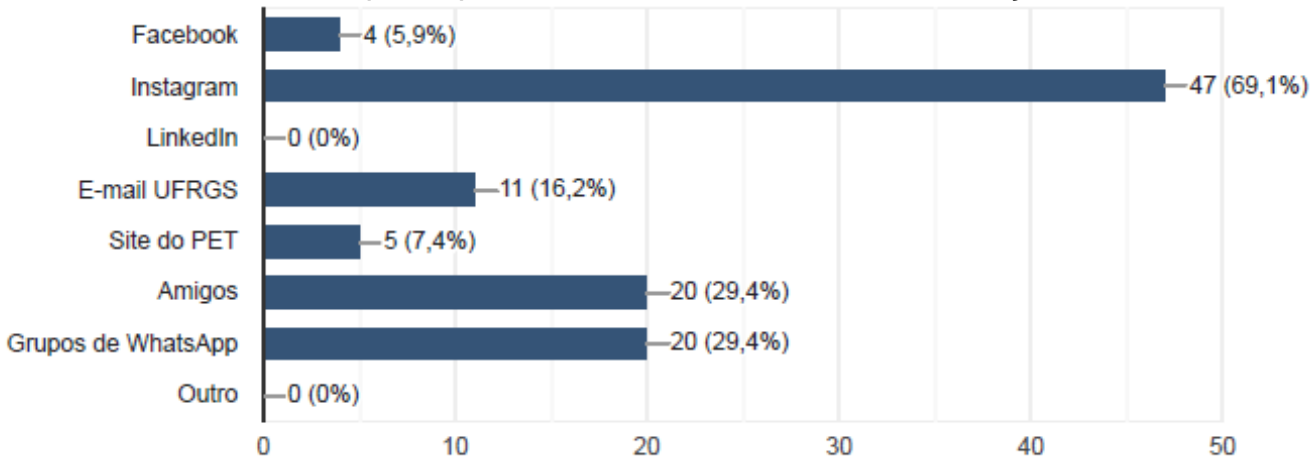

Figura 12 - Percepção dos participantes em relação ao curso de Excel

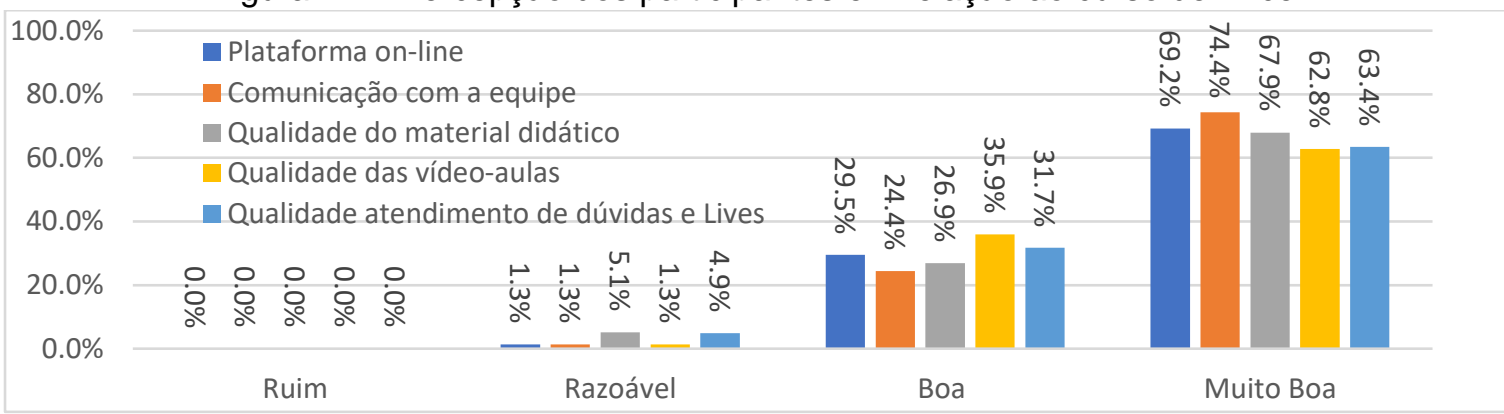

Em relação aos cursos, verificou-se que através da aplicação do questionário (feedback), a percepção dos participantes acerca dos cursos ministrados. A figura 12 mostra a percepção dos alunos quanto ao curso de Excel, sendo adotada uma escala qualitativa (ruim, razoável, bom e muito bom). Neste sentido, os participantes classificaram o curso como bom a muito bom, com uma média de $29,7 \%$ e $67,5 \%$, respectivamente, em todos os quesitos pesquisados, levando em consideração a plataforma digital, comunicação e atendimento, material didático (apostila e exercícios) e videoaulas.

Para o curso de Revit, que foi ministrado posteriormente, adotou-se uma escala quantitativa mais ampla, de 0 (péssimo) a 10 (ótimo). Em relação a percepção do curso (plataforma on-line, didática dos professores, atendimento e desafio - atividade final) a percepção foi bastante positiva (figura 13), sendo que o maior percentual de notas foi 10 , e mais de $95 \%$ das notas acima de 8 . Procurou-se verificar qual a percepção dos participantes quanto a qualidade das videoaulas (figura 14). De uma forma geral, verifica- 
se que a objetividade e os roteiros das videoaulas foram os itens que mais pontuaram. Quanto a qualidade de vídeo e áudio, o curso recebeu algumas críticas quanto a qualidade do áudio (principalmente, quanto a variação do volume ao longo dos vídeos). Quanto a este aspecto, cabe lembrar que as videoaulas não foram gravadas em estúdio, com utilização de equipamentos profissionais, apesar de passarem por uma edição antes de serem disponibilizados. Mas apesar disso, a percepção dos participantes quanto ao curso foi bastante positiva.

Figura 13 - Notas atribuídas em relação a percepção geral dos participantes em relação ao curso de Revit $100.0 \%$

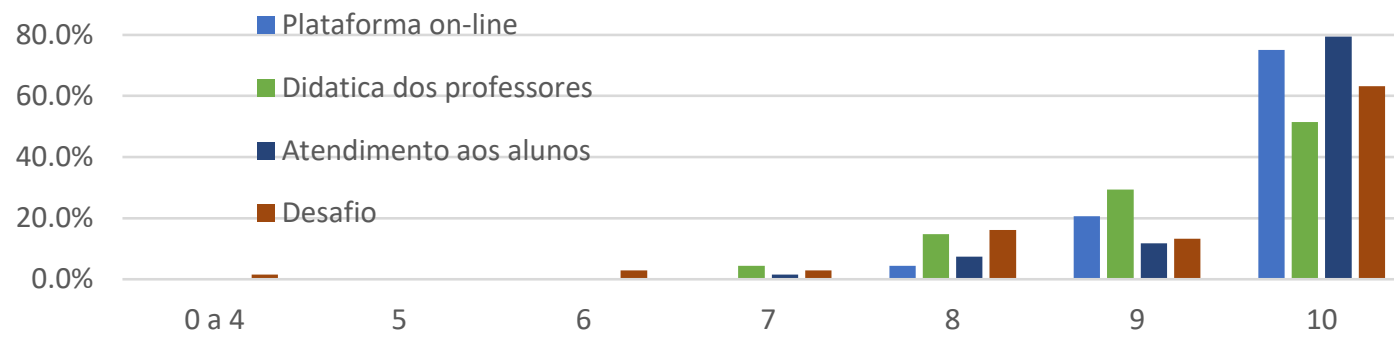

Figura 14 - Notas atribuídas em relação a percepção em relação as videoaulas dos participantes em relação ao curso de Revit

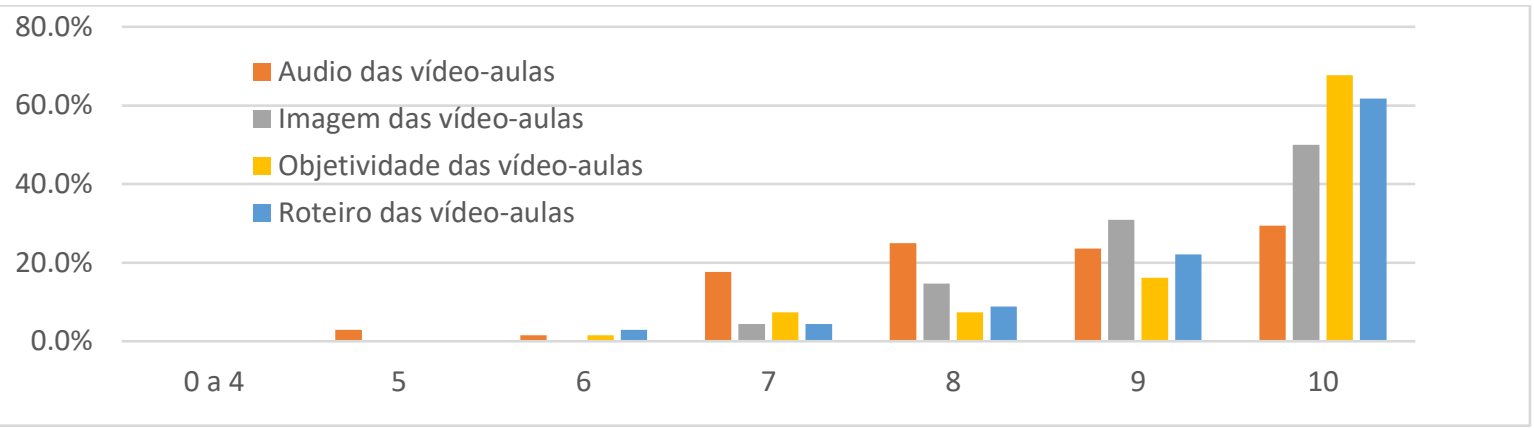

Figura 15: (a) melhores ferramentas de aprendizagem utilizadas pelos participantes e (b) uso da videoaulas

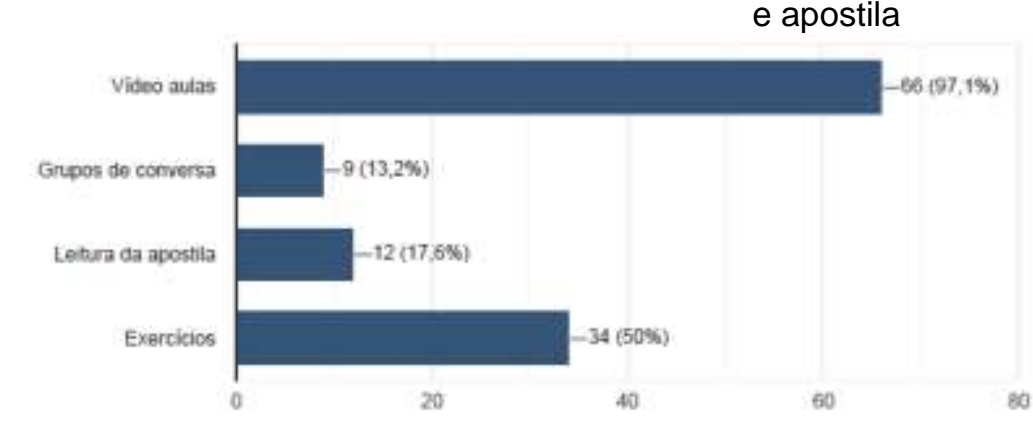

(a)

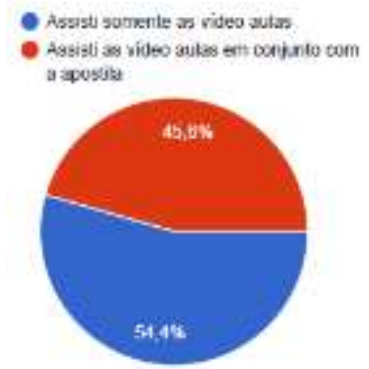

(b)

Ainda, quanto ao curso de Revit, a figura 15 mostra as ferramentas de ensino utilizadas pelos participantes ao longo do curso. Verifica-se que quase todos qualificaram as videoaulas $(97,1 \%)$ como a melhor ferramenta, seguido dos exercícios $(50 \%)$ e apostila $(17,6 \%)$. Chama atenção o fato da apostila ter sido apontada com pouca funcionalidade para o curso, uma vez que $45,6 \%$ dos participantes fez o curso utilizando as videoaulas em conjunto com a apostila. Isso demostra que no ambiente on-line, em se tratando de curso 
de software, as videoaulas que têm sido bastante aceitas pelos participantes, em detrimento ao material escrito (apostila).

\section{CONSIDERAÇÕES FINAIS}

O presente trabalho demostrou a capacidade de adaptação ao sistema remoto do Grupo PET Engenharia Civil, passando a oferecer curso de software de curta duração na modalidade on-line. Os resultados demostram uma ampliação no número de participantes de ambos os cursos (165\% no Excel e $157 \%$ no Revit). Ainda, ampliou-se o público atendido abrangendo alunos de ensino médio até profissionais com pós-graduação, bem como alunos de diversos cursos de graduação, no caso do Excel. Além disso, os cursos alcançaram participantes de 14 estados (incluindo o RS). Do ponto de vista socioeconômico, verifica-se que independentemente da renda e do número de pessoas que residem com os participantes, a taxa de computadores ( $\mathrm{PC}$ ou notebook), na média é inferior a uma unidade, mesmo para um público acadêmico e profissional. Os cursos receberam conceitos positivos, considerando a qualidade dos materiais (videoaulas, apostila, lista de exercícios e desafio), qualidade da plataforma on-line e atendimento durante os cursos. Dentre as ferramentas de ensino, os participantes quase foram unanimes em julgaram que as videoaulas como melhores $(97,1 \%)$, em detrimento a apostila que $17,6 \%$, demostrando que os cursos online ficam mais atrativos quando utilizam recursos audiovisuais.

\section{REFERÊNCIAS}

ADAMOPOULOS, P. What makes a great MOOC? An interdisciplinary analysis of student retention in on-line courses. In: INTERNATIONAL CONFERENCE ON INFORMATION SYSTEMS (ICIS), 34., 2013, Milan. Proceedings... Milan: Association of Information Science.

Disponível

em:

http://people.cs.pitt.edu/ phuongpham/comp/wiebe/Adamopoulos2013.pdf. Acessado em 08/04/2021.

AGONÁCS, N.; MATOS, J. F. Os Cursos On-line Abertos e Massivos (Mooc) como ambientes heutagógicos. Revista Brasileira de Estudos Pedagógicos. Brasília, v. 101, n. 257, p. 17-35, 2020.

BRASIL. Portaria MEC no 343, de 17/03/2020. Brasília, 2020a;

BRASIL. Medida Provisória no 919, de 30/01/2020. Brasília, 2020b.

FALCÃO. L. V.; JUCÁ, A. L.; VIEIRA, S. G.; ALVES, C. K. de A. A terapia ocupacional na atenção primária a saúde reinventando ações no cotidiano frente as alterações provocadas pelo COVID-19. Revista Interinstitucional Brasileira de Terapia Ocupacional. v.4, n. 3, p. 333-350, 2020.

MONDINI, V. E. D.; DOMINGUES, M. J. C, de S. Gestão da retenção de alunos em cursos on-line sob a perspectiva da aceitação da tecnologia. Revista Brasileira de Educação, v. 23, e230050, p. 1-29, 2018

RODRIGUES, M. A. Física na quarentena: resultados preliminares de um curso de extensão on-line. In: I Simpósio Sul-Americano de Pesquisa em Ensino de Ciências (SSAPEC), 2020, Cerro Largo, Anais. Cerro Largo. Disponível em: https://portaleventos.uffs.edu.br/index.php/SSAPEC/article/view/14417/9734. Acessado em 08/04/2021. 
UNIVERSIDADE FEDERAL DO RIO GRANDE DO SUL. Portaria no 1433, de 12/03/2021. Porto Alegre, 2021.

UNIVERSIDADE FEDERAL DO RIO GRANDE DO SUL. Portaria no 2286, de 17/03/2020. Porto Alegre, 2020a.

UNIVERSIDADE FEDERAL DO RIO GRANDE DO SUL. Portaria no 2291, de 17/03/2020. Porto Alegre, 2020b.

\title{
ANALYSIS OF THE PERCEPTION OF ON-LINE SOFTWARE COURSES (EXCEL AND REVIT) OFFERED DURING THE PANDEMIC PERIOD
}

\begin{abstract}
The PET Civil Engineering Group of UFRGS, among its several proposals to improve the graduate course, has promoted software courses of interest to students and professionals in the area, among which Excel and Revit can be highlighted. These are traditionally offered in person, but due to the pandemic corona virus, they had to be reformulated and administered online. This new context expanded the courses, transforming them into MOOC courses (massive, open and free) and also began to be treated as extension actions. At the end of the courses, participants are asked to complete a feedback questionnaire (prerequisite for receiving the certificate) of course evaluation. Thus, the objective of this work is to show the results of the evaluations of the first online edition of these courses, being analyzed data such as: (a) scope and scope, (b) socioeconomic and (c) quality and learning in the courses. It was found that the number of graduates was significantly increased (in the order of 160\%). It covered high school students to professionals with graduate degrees, including from other states and several courses, not being restricted to civil engineering undergraduates at UFRGS. From the socioeconomic point of view, it was found that the participants have an average computer/person rate of less than one, regardless of family income and number of people living with the participant. The evaluation of the course in terms of the quality of the material (video classes, exercises, handouts) and attendance was considered satisfactory, with concepts from good to very good and grades ranging from 8 to 10 .
\end{abstract}

Keywords: Online courses. Excel and Revit software. Course Evaluation 